\title{
Left Dorsomedial Frontal Brain Damage Is Associated with Insomnia
}

\author{
Michael Koenigs, ${ }^{1}$ Jessica Holliday, ${ }^{1}$ Jeffrey Solomon, ${ }^{2}$ and Jordan Grafman ${ }^{3}$ \\ ${ }^{1}$ Department of Psychiatry, University of Wisconsin-Madison, Madison, Wisconsin 53719, ${ }^{2}$ Medical Numerics, Inc., Germantown, Maryland 20876, and \\ ${ }^{3}$ Cognitive Neuroscience Section, National Institutes of Neurological Disorders and Stroke, National Institutes of Health, Bethesda, Maryland 20892
}

Insomnia is a common sleep disorder, yet its pathophysiological basis remains poorly understood. Studying a group of 192 patients with focal brainlesions, we show a significant association betweeninsomnia and left dorsomedial prefrontal damage. Ourfindings are the first todemonstratealink between insomnia and a discrete locus of brain damage, providing novel insight into the neurobiological mechanisms of sleep maintenance.

\section{Introduction}

Insomnia is a sleep disorder involving difficulty initiating and maintaining sleep, with associated detriments in mood and cognitive function during wakefulness. Despite the remarkable prevalence-sleep difficulty afflicts more than one in three adults (Ohayon and Reynolds, 2009)_and frequent association with serious mental health disorders such as anxiety and depression (Ohayon, 2009), the neurobiological underpinnings of insomnia remain poorly understood. Although sleep onset and maintenance is characterized by widespread changes in brain activity (Braun et al., 1997; Massimini et al., 2004), recent evidence suggests that left dorsal and medial frontal areas may be especially important in mediating sleep. One study using magnetoencephalography localized the greatest activity increases during both rapid eye movement (REM) and deep non-REM sleep to left dorsomedial prefrontal cortex (dmPFC) (Ioannides et al., 2009), whereas a second study using high-density electroencephalography (hd-EEG) found that sleep slow waves preferentially originate in the left frontoinsular area and cingulate gyrus (Murphy et al., 2009). These results suggest that left medial prefrontal cortex ( $\mathrm{mPFC})$ /insula may play a critical role in maintaining sleep, and by extension, insomnia. However, the correlative nature of the aforementioned neuroimaging data precludes any direct causal inference regarding the neural substrates of sleep initiation and maintenance. If left $\mathrm{mPFC} /$ insula is indeed critical for sleep initiation and maintenance, then damage to this area should be associated with insomnia. To test this prediction, we assessed the prevalence of insomnia in a large sample of individuals with focal brain lesions.

Received July 19, 2010; revised Sept. 14, 2010; accepted 0ct. 15, 2010.

This work was supported by the National Institute of Neurological Disorders and Stroke intramural research program. We thankSandi Bonifant for VHIS data management, Dr. Vanessa Raymont for psychiatric evaluations, and Dr. Jose Maisog for developing the $\chi^{2}$ false discovery rate software. We thank the veterans for their participation in the VHIS.

Correspondenceshould be addressed to either of the following:Michael Koenigs, Department of Psychiatry, University of Wisconsin-Madison, 6001 Research Park Boulevard, Madison, WI 53719, E-mail: mrkoenigs@wisc.edu; or Jordan Grafman, Cognitive Neuroscience Section, National Institute of Neurological Disorders and Stroke, National Institutes of Health, 10 Center Drive, Bethesda, MD 20892, E-mail: grafmanj@ninds.nih.gov.

DOl:10.1523/JNEUROSCI.3745-10.2010

Copyright $\odot 2010$ the authors $\quad 0270-6474 / 10 / 3016041-03 \$ 15.00 / 0$

\section{Materials and Methods}

Participants. Participants were drawn from the Phase 3 Vietnam Head Injury Study (VHIS) registry, which includes American male veterans who suffered brain damage from penetrating head injuries in the Vietnam War $(n=199)$. All subjects gave informed written consent. Phase 3 testing occurred between April 2003 and November 2006.

Lesion analysis. CT data were acquired during the Phase 3 testing period. Axial CT scans without contrast were acquired at Bethesda Naval Hospital on a GE Medical Systems Light Speed Plus CT scanner in helical mode ( $\sim 150$ slices per subject, field of view covering head only). Images were reconstructed with an in-plane voxel size of $0.4 \times 0.4 \mathrm{~mm}$, overlapping slice thickness of $2.5 \mathrm{~mm}$, and a $1 \mathrm{~mm}$ slice interval. Lesion location and volume were determined from CT images using the Analysis of Brain Lesion software (Makale et al., 2002; Solomon et al., 2007) contained in MEDx v3.44 (Medical Numerics) with enhancements to support the Automated Anatomical Labeling atlas (Tzourio-Mazoyer et al., 2002). Lesion volume was calculated by manual tracing of the lesion in all relevant slices of the CT image then summing the traced areas and multiplying by slice thickness. A trained neuropsychiatrist performed the manual tracing, which was then reviewed by J.G., who was blind to the results of the neuropsychological testing. As part of this process, the CT image of each subject's brain was spatially normalized to a CT template brain image. This template was created by spatial normalization of a neurologically healthy individual's CT brain scan to MNI space (Collins et al., 1994) using the Automated Image Registration program (Woods et al., 1993). For each subject, a lesion mask image in MNI space was saved for voxel-based lesion-symptom analysis (Bates et al., 2003).

Insomnia self-report. From the VHIS sample, 192 brain-injured participants underwent CT brain imaging and completed the Hamilton Anxiety Rating Scale (HAM-A) (Hamilton, 1959). The HAM-A includes one item specifically related to insomnia-the subject rates his difficulty falling asleep or staying asleep on a scale from 0 to 4 , with higher scores indicating more severe insomnia. To evaluate whether insomnia is associated with damage to specific brain areas, we examined the lesion locations of those subjects who reported moderate or severe insomnia (scores of 2 or greater on the HAM-A insomnia item; $n=27$ ).

\section{Results}

As can be seen in Figure 1 (third row), the most common area of damage among the individuals with moderate-to-severe insomnia was the left dmPFC. To test for statistical significance, we computed a map of $\chi^{2}$ values, where the value of each voxel represents the $\chi^{2}$ statistic comparing the frequency of moderate-to-severe insomnia 

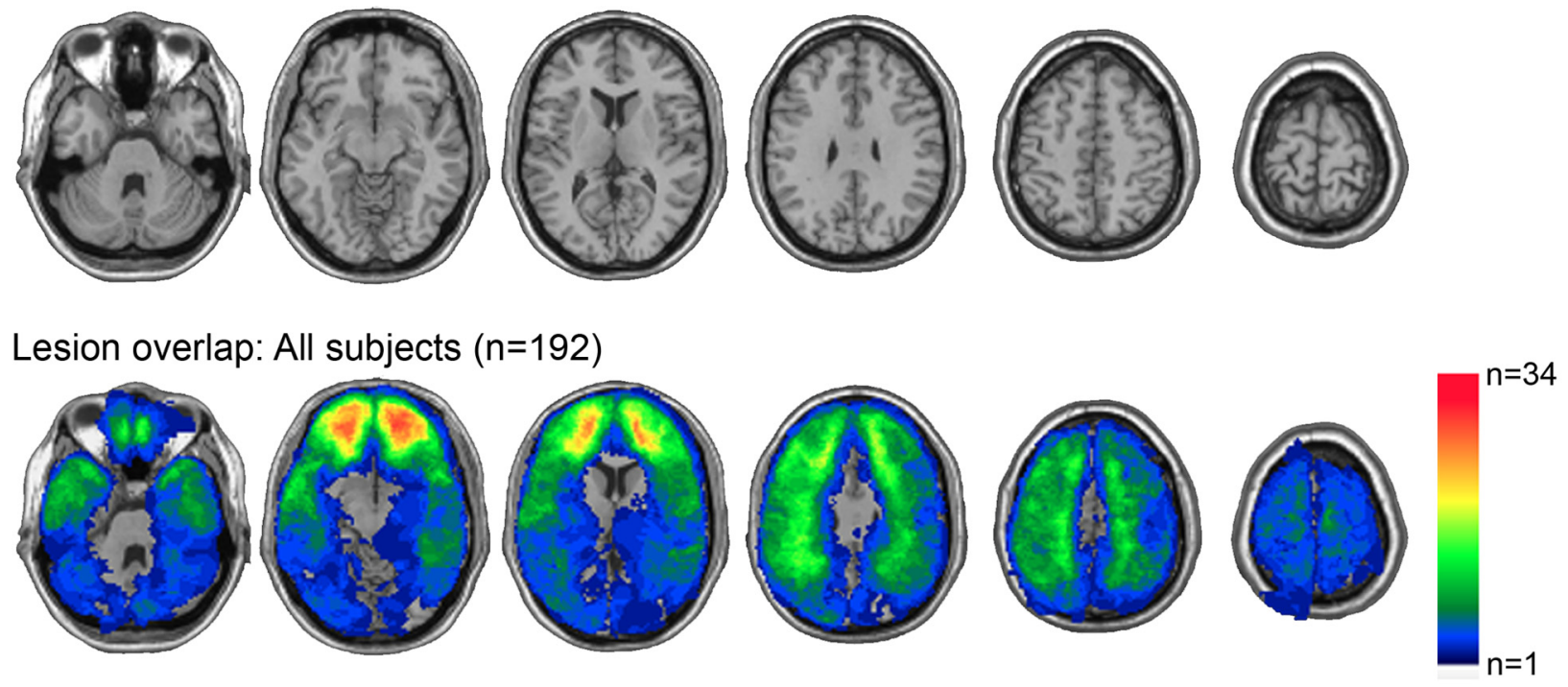

Lesion overlap: Subset of subjects with insomnia $(n=27)$
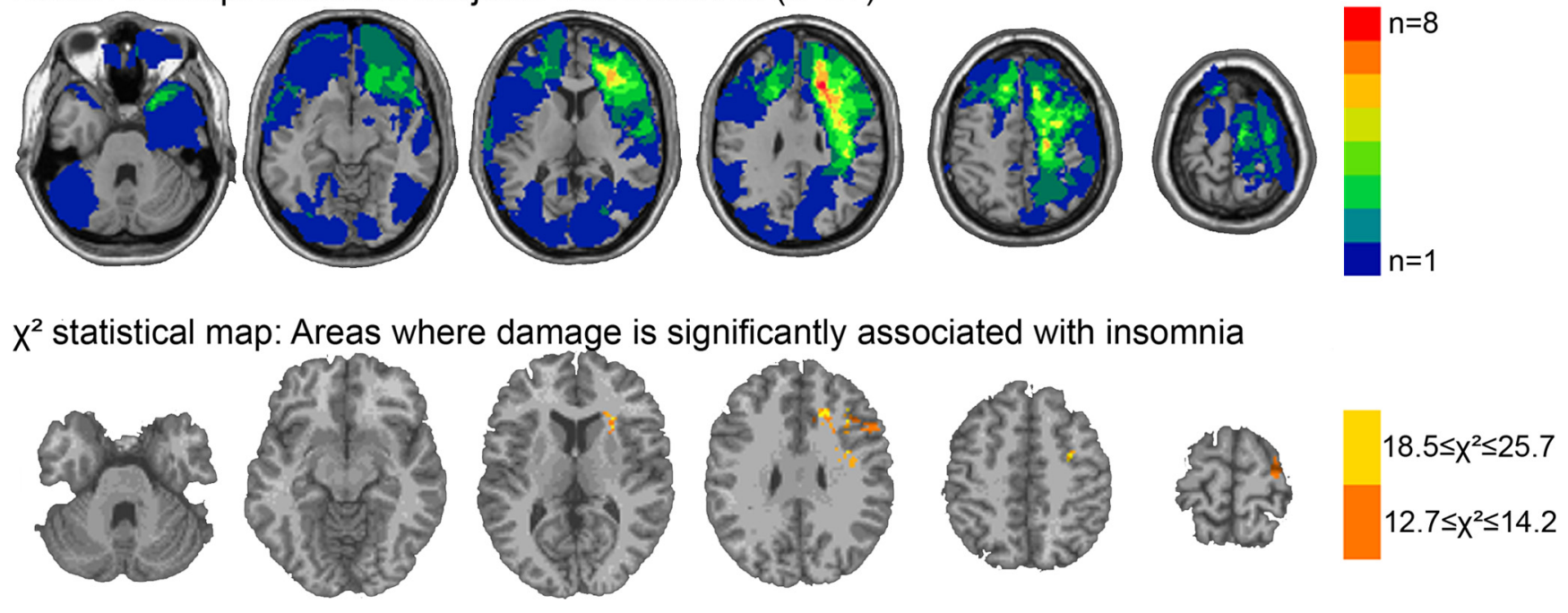

Figure 1. Left dmPFC damage is associated with insomnia. Top row, Transverse slices of a healthy adult brain, for reference. Corresponding slices are shown in rows $2-4$. In all slices, the left hemisphere is on the reader's right. Second row, Lesion overlap of all subjects $(n=192)$ who completed the HAM-A and CT imaging. The color bar indicates the number of overlapping lesions at each voxel. Maximal overlap occurs in ventral prefrontal cortex bilaterally. Third row, Lesion overlap of the subset of subjects reporting moderate-to-severe insomnia. The color bar indicates the number of overlapping lesions at each voxel. Maximal overlap occurs in left dmPFC. Bottom row, $\chi^{2}$ statistical map. The color bar indicates $\chi^{2}$ values that exceed the threshold for statistical significance at a given voxel (corrected for multiple comparisons). The most significant $\chi^{2}$ values are found in left dmPFC.

among individuals with damage to that voxel to the frequency of moderate-to-severe insomnia among individuals without damage to that voxel. To determine the critical $\chi^{2}$ value for statistical significance, we used a false discovery rate correction for multiple comparisons with $q=0.05$ (Genovese et al., 2002), which resulted in a critical $\chi^{2}$ value of 12.6 . This analysis revealed statistically significant voxels in left dmPFC and adjacent areas (Fig. 1, fourth row).

These results demonstrate that damage to left dmPFC is associated with insomnia. But given that insomnia is a common symptom of mood and anxiety disorders, it is possible that the observed association between insomnia and left dmPFC damage is secondary to the role of this brain area in regulating mood and anxiety. In other words, the identification of left dmPFC in Figure 1 may be due to the selection of patients with heightened levels of depression and/or anxiety symptoms in general, rather than due to the selection of patients with insomnia, in particular. To examine this possibility, we selected a subset of veterans based on all the individuals from the no insomnia group with depression and anxiety symptoms that were equal to
Table 1. Group characteristics

\begin{tabular}{lccl}
\hline & $\begin{array}{l}\text { Insomnia } \\
(n=27)\end{array}$ & $\begin{array}{l}\text { No insomnia } \\
(n=172)\end{array}$ & $\begin{array}{l}\text { No insomnia: high } \\
\text { depression/anxiety } \\
\text { subset }(n=27)\end{array}$ \\
\hline Age & $58.0(2.3)$ & $58.3(3.2)$ & $57.5(2.6)$ \\
IQ & $101.7(15.7)$ & $102.5(14.7)$ & $97.4(10.7)$ \\
General memory & $96.7(12.8)$ & $98.1(16.3)$ & $92.8(15.9)$ \\
PTSD prevalence & 0.48 & 0.08 & 0.48 \\
BDI-II & $16.1(12.2)$ & $8.2(8.0)$ & $20.9(10.2)$ \\
STAl trait anxiety & $61.0(14.4)$ & $52.1(10.5)$ & $63.7(12.7)$ \\
Lesion volume $\left(\mathrm{cm}^{3}\right)$ & $40.7(43.4)$ & $40.3(43.4)$ & $40.4(46.5)$ \\
\hline
\end{tabular}

Age, mean (SD). IQ, mean (SD); full-scale IO from Wechsler Adult Intelligence Scale-III (Wechsler, 1997a). General memory, mean (SD); general memory index from Wechsler Memory Scales-III (Wechsler, 1997b). Posttraumatic stress disorder (PTSD) prevalence, proportion of patients diagnosed with current PTSD based on a psychiatrist's evaluation using the Structured Clinical Interview for DSM-IV Axis I disorders (First, 2002). BDI-II, mean (SD) total Beck Depression Inventory-II score (Beck et al., 1996). STAl, mean (SD) trait anxiety scaled score from State-Trait Anxiety Inventory (Spielberger et al., 1970). Lesion volume, mean (SD) lesion volume in cubic centimeters. 

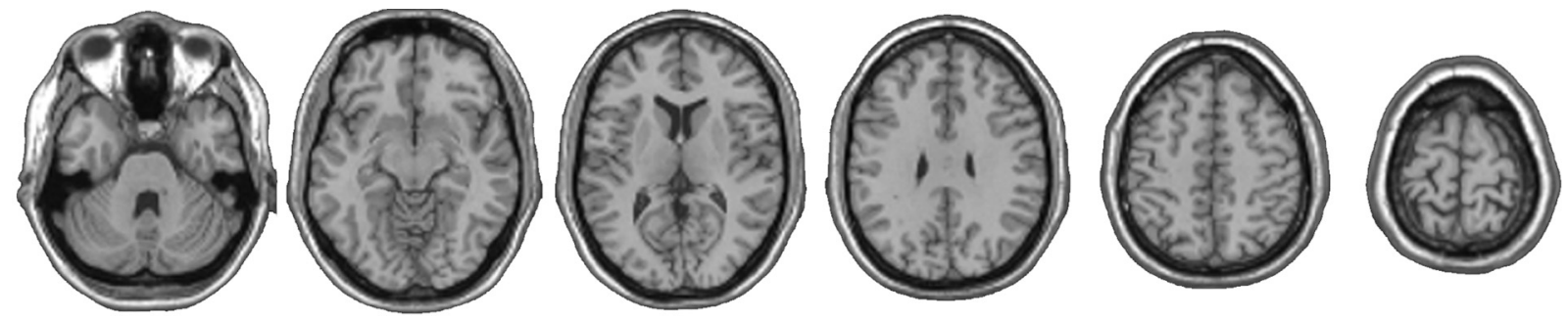

Lesion overlap: Subset of subjects with no insomnia but high overall depression/anxiety $(n=27)$
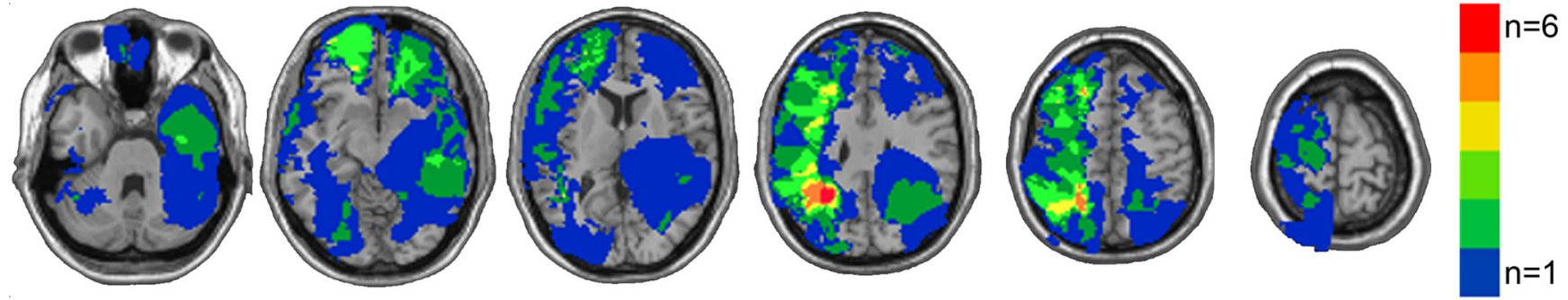

Figure 2. Overall high levels of depression/anxiety symptoms are not associated with left dmPFC damage. Top row, Transverse slices of a healthy adult brain, for reference. In all slices, the left hemisphere is on the reader's right. Bottom row, Lesion overlap of subjects $(n=27)$ with significant overall levels of mood/anxiety symptoms but no insomnia. The color bar indicates the number of overlapping lesions at each voxel. There are no multiple overlapping lesions in left dmPFC in this group.

or greater than the symptoms observed in the insomnia group (Table 1). The lesion distribution of this subgroup $(n=27)$ (Fig. 2$)$ indicates no multiple overlapping lesions in left dmPFC. Hence, the concentration of left dmPFC lesions observed in Figure 1 is not simply a result of selecting patients with generally high levels of mood and anxiety symptoms, and we therefore conclude that the identification of left dmPFC damage in the insomnia group is specifically due to an association between left dmPFC damage and sleep disturbance.

\section{Discussion}

The present results join with previous sleep research to suggest a possible mechanism by which left $\mathrm{dmPFC}$ lesions impair sleep. Electrophysiological neuroimaging data indicate that sleep slow waves preferentially originate in left insula and propagate posteriorly along the cingulate (Murphy et al., 2009). Thus, lesions located superior and medial to the left insula (precisely the area identified in this study), could significantly disrupt the propagation of sleep slow waves along the left insula-cingulate corridor, resulting in difficulty initiating or maintaining sleep. In the present study, we are unable to test this hypothesis directly, as our sleep measure was limited to subjects' self-report of insomnia. However, future studies of focal dmPFC dysfunction (as with stroke patients or transcranial brain stimulation), coupled with more sophisticated measures of sleep maintenance (such as hd-EEG sleep recording), could provide further converging evidence for the importance of left dmPFC in sleep slow-wave propagation.

To our knowledge, this is the first study demonstrating a link between insomnia and a discrete locus of brain damage. The lesion data presented here indicate a critical role for left dmPFC in mediating sleep.

\section{References}

Bates E, Wilson SM, Saygin AP, Dick F, Sereno MI, Knight RT, Dronkers NF (2003) Voxel-based lesion-symptom mapping. Nat Neurosci 6:448-450.

Beck AT, Steer RA, Brown GK (1996) Manual for the Beck Depression Inventory-II. San Antonio: Psychological Corporation.

Braun AR, Balkin TJ, Wesenten NJ, Carson RE, Varga M, Baldwin P, Selbie S, Belenky G, Herscovitch P (1997) Regional cerebral blood flow throughout the sleep-wake cycle: an H2(15)O PET study. Brain 120:1173-1197.
Collins DL, Neelin P, Peters TM, Evans AC (1994) Automatic 3D intersubject registration of MR volumetric data in standardized Talairach space. J Comput Assist Tomogr 18:192-205.

First MB, Spitzer RL, Gibbon M, Williams JBW (2002) Structured clinical interview for DSM-IV-TR Axis I disorders, research version, non-patient edition (SCID-I/NP). New York: Biometrics Research, New York State Psychiatric Institute.

Genovese CR, Lazar NA, Nichols T (2002) Thresholding of statistical maps in functional neuroimaging using the false discovery rate. Neuroimage $15: 870-878$

Hamilton M (1959) The assessment of anxiety states by rating. Br J Med Psychol 32:50-55.

Ioannides AA, Kostopoulos GK, Liu L, Fenwick PB (2009) MEG identifies dorsal medial brain activations during sleep. Neuroimage 44:455-468.

Makale M, Solomon J, Patronas NJ, Danek A, Butman JA, Grafman J (2002) Quantification of brain lesions using interactive automated software. Behav Res Methods Instrum Comput 34:6-18.

Massimini M, Huber R, Ferrarelli F, Hill S, Tononi G (2004) The sleep slow oscillation as a traveling wave. J Neurosci 24:6862-6870.

Murphy M, Riedner BA, Huber R, Massimini M, Ferrarelli F, Tononi G (2009) Source modeling sleep slow waves. Proc Natl Acad Sci U S A 106:1608-1613.

Ohayon MM (2009) Observation of the natural evolution of insomnia in the American general population cohort. Sleep Med Clin 4:87-92.

Ohayon MM, Reynolds CF 3rd (2009) Epidemiological and clinical relevance of insomnia diagnosis algorithms according to the DSM-IV and the International Classification of Sleep Disorders (ICSD). Sleep Med 10:952-960.

Solomon J, Raymont V, Braun A, Butman JA, Grafman J (2007) Userfriendly software for the analysis of brain lesions (ABLe). Comput Methods Programs Biomed 86:245-254.

Spielberger CD, Gorsuch RL, Lushene RE (1970) Manual for the State-Trait Anxiety Inventory. Palo Alto: Consulting Psychologists.

Tzourio-Mazoyer N, Landeau B, Papathanassiou D, Crivello F, Etard O, Delcroix N, Mazoyer B, Joliot M (2002) Automated anatomical labeling of activations in SPM using a macroscopic anatomical parcellation of the MNI MRI single-subject brain. Neuroimage 15:273-289.

Wechsler D (1997a) Wechsler Adult Intelligence Scale-III. San Antonio: The Psychological Corporation.

Wechsler D (1997b) Wechsler Memory Scale, third edition manual. San Antonio: The Psychological Corporation.

Woods RP, Mazziotta JC, Cherry SR (1993) MRI-PET registration with automated algorithm. J Comput Assist Tomogr 17:536-546. 